\title{
Stratified Scaffold Design for Engineering Composite Tissues
}

Christopher Z. Mosher ${ }^{1}$, Jeffrey P. Spalazzi ${ }^{1}$, and Helen H. Lu'

${ }^{1}$ Biomaterials and Interface Tissue Engineering Laboratory, Department of Biomedical Engineering, Columbia University, New York, New York 10027

Corresponding author: Helen H. Lu, Ph.D.

A: 351 Engineering Terrace, 1210 Amsterdam Avenue, New York, New York, 10027

$\mathrm{P}:+1$ 212-854-4071

E: hhlu@columbia.edu

\begin{abstract}
A significant challenge to orthopaedic soft tissue repair is the biological fixation of autologous or allogeneic grafts with bone, whereby the lack of functional integration between such grafts and host bone has limited the clinical success of anterior cruciate ligament $(A C L)$ and other common soft tissue-based reconstructive grafts. The inability of current surgical reconstruction to restore the native fibrocartilaginous insertion between the ACL and the femur or tibia, which minimizes stress concentration and facilitates load transfer between the soft and hard tissues, compromises the longterm clinical functionality of these grafts. To enable integration, a stratified scaffold design that mimics the multiple tissue regions of the $A C L$ interface (ligament-fibrocartilage-bone) represents a promising strategy for composite tissue formation. Moreover, distinct cellular organization and phase-specific matrix heterogeneity achieved through co- or tri-culture within the scaffold system can promote biomimetic multi-tissue regeneration. Here, we describe the methods for fabricating a tri-phasic scaffold intended for ligament-bone integration, as well as the tri-culture of fibroblasts, chondrocytes, and osteoblasts on the stratified scaffold for the formation of structurally contiguous and compositionally distinct regions of ligament, fibrocartilage and bone. The primary advantage of the tri-phasic scaffold is the recapitulation of the multi-tissue organization across the native interface through the layered design. Moreover, in addition to ease of fabrication, each scaffold phase is similar in polymer composition and therefore can be joined together by sintering, enabling the seamless integration of each region and avoiding delamination between scaffold layers.
\end{abstract}

Keywords: biological fixation; multi-phased scaffold; co-culture; enthesis; anterior cruciate ligament; interface tissue engineering

\section{Introduction}

The anterior cruciate ligament (ACL) is the most commonly injured knee ligament [1], with approximately 100,000 reconstructive surgeries performed annually in the United States [1-5] to restore the ligament's function as the primary knee joint stabilizer. The ACL's lack of vascularity and poor healing potential [6] make surgical intervention necessary, where autologous bone-patellar tendon-bone (BPTB) and hamstring tendon grafts are often used to rebuild the ligament. Hamstring tendon-based grafts are increasingly used as a replacement for BPTB grafts, which have been reported to result in severe donor site morbidity $[7,8]$.

The long-term performance of hamstring tendon grafts is a measure of the structural and material properties of the graft, intra-articular positioning [9,10], initial graft tension [11-16], and post-operative rehabilitation. Here, we focus on the graft's potential as a function of its fixation $[17,18]$ in the joint space. Although mechanical graft fixation has been improved clinically through the use of transfemoral pins and screws, biological fixation with subchondral bone remains a limiting factor to clinical success [17-19]. With current ACL reconstructionmethods, the native fibrocartilage insertion site fails to regenerate $[20,20]$ and without this functional interface, biological graft-bone fixation cannot be achieved [17-19,21-23].

The ACL, and other soft tissues with direct insertions into bone, exhibit three distinct yet continuous regions with controlled variation in cell type and matrix heterogeneity: ligament, fibrocartilage, and bone [24-32]. The ligament proper is composed of fibroblasts embedded in a type I and II collagen matrix. Within the fibrocartilagenous region, nonmineralized (ovoid chondrocytes, type II collagen, and proteoglycan-rich matrix) and mineralized (hypertrophic chondrocytes in a calcified matrix [30,30] containing type $X$ collagen [28]) fibrocartilage zones are observed. Lastly, 
the subchondral bone consists of osteocytes, osteoblasts and osteoclasts within a mineralized type I collagen matrix. The method for tri-phasic scaffold fabrication outlined below aims to mimic this control over matrix heterogeneity in order to recapitulate the multi-tissue ligament-bone transition. The scaffold is thus designed to support interface regeneration that will facilitate load transfer between soft and hard tissues, minimizing stress concentration [25,33] and enabling graft-bone integration. Moreover, seeding of the scaffold layers with fibroblasts, osteoblasts, and chondrocytes has demonstrated spatial control over cell and matrix distribution [34,35], which may be translated to other interface tissue engineering or composite tissue regeneration strategies.

\section{Materials}

\subsection{Scaffold Preparation}

2.1a Scaffold Phase A:

Polyglactin 10:90 Knitted Mesh Sheets (Vicryl VKML, Ethicon, Somerville, NJ), 5x5 mm squares

2.1b Scaffold Phase B

Poly(D-L-lactide-co-glycolide) 85:15 copolymer (PLGA, $\mathrm{M}_{\mathrm{w}} \approx 123.6 \mathrm{kDa}$, Alkermes, Cambridge, MA)

Dichloromethane (DCM, EM Science, Gibbstown, NJ)

Polyvinyl alcohol (PVA, $\mathrm{M}_{\mathrm{w}} \approx 89 \mathrm{kDa}$, Sigma, St. Louis, MO)

2.1c Scaffold Phase $C$

Poly(D-L-lactide-co-glycolide) 85:15 copolymer (PLGA, $\mathrm{M}_{\mathrm{w}} \approx 123.6 \mathrm{kDa}$, Alkermes, Cambridge, MA)

45S5 bioactive glass (BG, $20 \mu \mathrm{m}$, Mo-Sci Corp., Rolla, MD)

Dichloromethane (DCM, EM Science, Gibbstown, NJ)

2.1d Sintering and Sterilization of Scaffold Phases

Ethylene oxide (Sigma, St. Louis, MO)

\subsection{Cell Culture and Seeding}

Bovine Knee Joints (Green Village Packing Co, Green Village, NJ)

\#22 scalpel blade (Thermo Fisher Scientific Inc., Waltham, MA)

Phosphate-buffered saline (PBS, Sigma Chemicals, St. Louis, MO): One packet PBS powder into $1 \mathrm{~L}$ deionized water Fully supplemented (F/S) culture media: Dulbecco's modified Eagle medium with 10\% fetal bovine serum, 1\% nonessential amino acids, and 1\% penicillin/streptomycin (Mediatech, Herndon, VA)

Digestion media: F/S culture media with $10 \%$ penicillin/streptomycin instead of $1 \%$, and the addition of $1 \%$ ampicillin B, $1 \%$ gentamicin, and 10\% collagenase II solution (Worthington Biochemical Corp., Lakewood, NJ) (see Note 1)

Cell culture grade agarose (Sigma Chemical, St. Louis, MO)

\section{Methods}

Carry out the following procedures at room temperature (non-sterile).

\subsection{Scaffold Phase Fabrication}

1. Phase A (see Figure 2A):

- $\quad$ Sinter square segments of Vicryl VKML sheets into cylindrical molds at $150^{\circ} \mathrm{C}$ for $20 \mathrm{hrs}$ under a $1.6 \mathrm{~N}$ load.

2. Phase B (see Figure 2B):

- $\quad$ Form PLGA microspheres via water/oil/water emulsion [36]. First, dissolve PLGA in 10\% w/v DCM.

- Slowly pour this solution into 1\% PVA mixing at 200 RPM to form microspheres. Allow the microspheres to harden for $20 \mathrm{hrs}$, then recover by filtration, rinse with deionized water, and air dry.

- $\quad$ A microsphere yield of $\sim 90 \%$ the original PLGA mass should be achieved.

- $\quad$ Sinter the resulting microspheres above the polymer glass transition temperature at $55^{\circ} \mathrm{C}$ in an oven for 5 hrs in a cylindrical mold under a $1.1 \mathrm{~N}$ load to form Phase B of the scaffold.

3. Phase C (see Figure 2C):

Fabricate composite 4:1 PLGA:BG microspheres.

- $\quad$ Dissolve $1 \mathrm{~g}$ PLGA in $15 \mathrm{~mL}$ DCM by vortexing for $1 \mathrm{hr}$.

- $\quad$ Suspend $0.25 \mathrm{~g} \mathrm{BG}$ in the solution by vortexing for $10 \mathrm{~min}$. Let the solution sit at room temperature for 20 $\min$.

- $\quad$ Slowly pour into 1\% PVA while mixing at 225 RPM. Mix for 4 hrs and rinse with 4 L deionized water. 
- $\quad$ Dry the microspheres at room temperature for $24 \mathrm{hrs}$ in a fume hood. A yield of $70 \%$ the original PLGA and BG total mass should be achieved.

- $\quad$ Sinter the resulting microspheres at $55^{\circ} \mathrm{C}$ in an oven for $5 \mathrm{hrs}$ in a cylindrical mold under a $1.1 \mathrm{~N}$ load to form Phase $\mathrm{C}$ of the scaffold.

4. Integrate the three scaffold phases by sequentially inserting each phase into a cylindrical mold followed by a mesh disc, and sintering the 5 layers for $10 \mathrm{hrs}$ at $55^{\circ} \mathrm{C}$ under a $1.1 \mathrm{~N}$ load, above the glass transition temperature of PLGA (see Figure 1).

5. Sterilize the resulting tri-phasic scaffold with ethylene oxide and vacuum desiccate in a sterile environment for at least 3-5 days prior to cell seeding.

Carry out all remaining procedures at room temperature under sterile conditions, unless otherwise specified.

\subsection{Cell Culture}

1. Wash neonatal bovine knee joints in soapy water for $20 \mathrm{~min}$, followed by a $40 \mathrm{~min}$ wash in $70 \%$ ethanol to sterilize.

2. Excise the patella and meniscus to expose the cruciate ligaments, and harvest the mid-section of the ACL. Mince ligament tissue with a \#22 scalpel blade and submerge the pieces in F/S culture media (see Note 2). Monitor cell migration from the explant, and only use cells derived from the second migration to ensure a homogenous fibroblast population $[37,38]$.

3. Harvest articular cartilage from the distal femur and proximal tibia with a scalpel blade, taking care not to violate the subchondral bone. Isolate primary bovine chondrocytes from the cartilage through collagenase digestion in digestion culture media.

4. Isolate cortical bone chips from bovine tibiae using a bone ronguer. Rinse the bone chips thoroughly with PBS (see Notes 3 and 4) to remove bone marrow, and submerge them in F/S culture media. Only use osteoblasts from the second migration for tri-culture on the scaffold.

5. Expand fibroblasts and osteoblasts in F/S culture media, and passage them at least once. Plate chondrocytes in F/S culture media 3 days prior to seeding onto scaffolds. Incubate all the cells at $37^{\circ} \mathrm{C}$ under humidified conditions and $5 \% \mathrm{CO}_{2}$.

\subsection{Cell Seeding}

1. Pre-coat the wells of a 12-well plate with $250 \mu \mathrm{L} /$ well $2 \%$ sterile agarose in order to limit cell migration out of the scaffolds (see Note 5 and Note 6).

2. Load $5 \times 10^{5}$ chondrocytes/scaffold, in a suspension of $0.5 \%$ agarose, onto Phase B of the scaffolds (see Note 7 ).

3. Allow the agarose to gel for $15 \mathrm{~min}$.

4. Add osteoblasts to Phase $\mathrm{C}$ of the scaffolds at a density of $2.5 \times 10^{5}$ osteoblasts/scaffold (see Note 8 ).

5 . Allow the cells to adhere to the scaffold for $20 \mathrm{~min}$.

6. Add fibroblasts to Phase A of the scaffolds at a density of $5 \times 10^{5}$ fibroblasts/scaffold.

7. Allow the cells in tri-culture (see Note 9) to attach for $20 \mathrm{~min}$, followed by the addition of $3 \mathrm{~mL}$ F/S culture media in each well of the 12 -well plate.

8. Incubate the samples at $37^{\circ} \mathrm{C}$ under humidified conditions and $5 \% \mathrm{CO}_{2}$.

\section{Results}

Adherence to the proposed fabrication protocol produced a tri-phasic scaffold designed for multi-tissue integration via co- or tri-culture of cells within heterogeneous, stratified scaffold layers [34,35] (see Figure 2) where each phase displayed significantly different porosity characteristics and intrusion volumes post-fabrication, summarized in Table 1. The height and diameter of each phase was similar, while porosity and the resulting intrusion volume decreased from Phase $A$ to $B$ to $C$, This demonstrates the capability of the fabrication design to produce a structurally contiguous scaffold with distinct, integrated layers that mimic the transition between multiples tissue types.

Table 1. Summary of postfabrication characterization of triphasic scaffold phases, modified from Spalazzi et. al., 2006 [35].

\begin{tabular}{|l|l|c|c|l|l|l|}
\hline $\begin{array}{l}\text { Phas } \\
\text { e }\end{array}$ & Composition & $\begin{array}{c}\text { Height } \\
(\mathbf{m m})\end{array}$ & $\begin{array}{c}\text { Diameter } \\
(\mathbf{m m})\end{array}$ & Porosity (\%) & $\begin{array}{c}\text { Mode Pore } \\
\text { Diameter }(\boldsymbol{\mu m})\end{array}$ & $\begin{array}{c}\text { Intrusion } \\
\text { Volume }(\boldsymbol{\mu L})\end{array}$ \\
\hline A & $10: 90$ PLGA & $2.44 \pm 0.14$ & $6.75 \pm 0.12$ & $58 \pm 5$ & $73 \pm 11$ & $41 \pm 8$ \\
\hline
\end{tabular}




\begin{tabular}{|l|l|l|l|l|l|l|}
\hline B & $85: 15$ PLGA & $2.2 \pm 0.2$ & $7.32 \pm 0.08$ & $34 \pm 4$ & $75 \pm 7$ & $28 \pm 7$ \\
\hline C & $80 \% 85: 15$ PLGA, 20\% BG & $1.5 \pm 0.2$ & $6.5 \pm 0.4$ & $26.7 \pm 0.4$ & $62 \pm 3$ & $14.5 \pm 0.1$ \\
\hline
\end{tabular}

\section{Discussion and Conclusions}

Here, tri-culture of fibroblasts, chondrocytes, and osteoblasts within distinct regions of the scaffold is suggested to recapitulate the ligament-fibrocartilage-bone interface associated with ACL repair [34,35]. The scaffold design is broadly applicable in other areas of composite tissue engineering, and provides a useful tool for multi-tissue integration and functional repair. Additionally, the scaffold's ease of fabrication and seamless transition between tissue types makes it an attractive option in this field, where stratified designs facilitate multi-tissue healing. The use of sintering in this system is especially advantageous, where the same bulk polymer is utilized in each phase and a single glass transition temperature is exceeded to achieve successful sintering. Through this method, polymer chains in each phase rearrange simultaneously and promote integration of adjacent, stratified scaffold layers. The resulting scaffold contains heterogeneous regions devoid of delamination due to intermingling of polymer chains. While three distinct regions were produced in this study, many regions can be produced by this design to mimic the transition between multiple tissue types. Moreover, the composition of each phase can be manipulated to tailor local mechanical properties, as well as bulk mechanical properties of the scaffold. This design may be applied to a variety of tissue engineering applications, where a multi-tissue transitions are desired.

\section{Notes}

1. Dissolve 20x collagenase II into Dulbecco's modified eagle medium containing only antibiotics (no fetal bovine serum). Filter this with a syringe before adding to F/S culture media to achieve a final solution with $10 \%$ collagenase II.

2. Supplemented Culture media should be stored at $4^{\circ} \mathrm{C}$ when not in use, and may be stored for up to 1 month prior to use.

3. Filter PBS using a sterile filter with $0.22 \mu \mathrm{m}$ pores.

4. Sterile-filtered PBS can be stored in a glass container at room temperature for up to 6 months after filtering.

5. Liquefy agarose by microwaving, and allow the solution to cool/gel for 10-20 min at room temperature. The gelling temperature of agarose is $32-45^{\circ} \mathrm{C}$.

6. Post-fabrication diameter of scaffold phases A, B, and C described for cell seeding is approximately $6.5 \mathrm{~mm}, 6.9$ $\mathrm{mm}$, and $7.1 \mathrm{~mm}$, respectively, and the thickness for each phase measures $1.85 \mathrm{~mm}, 1.90 \mathrm{~mm}$, and $1.84 \mathrm{~mm}$, respectively.

7. For co-culture of osteoblasts and fibroblasts, skip steps 2 and 3.

8. The fibroblast-to-osteoblast ratio was $2: 1$ due to the higher surface area of Phase $A$ as determined by mercury porosimetry.

9. For tri-culture, the primary cells used are typically between passage 2 and 5 . 


\section{Figures}

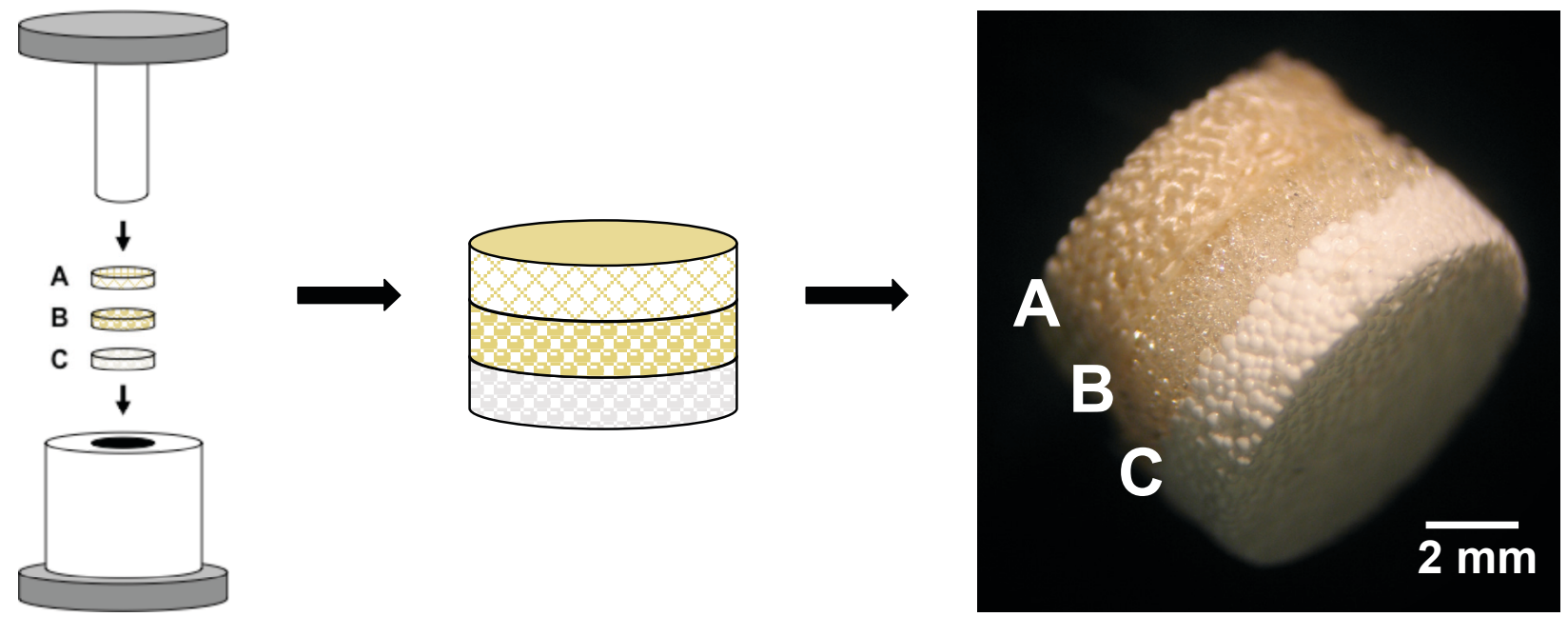

Figure 1. Tri-phasic scaffold fabrication. A, B, and C correspond to the phases listed under section 3.1 Scaffold Phase Fabrication.

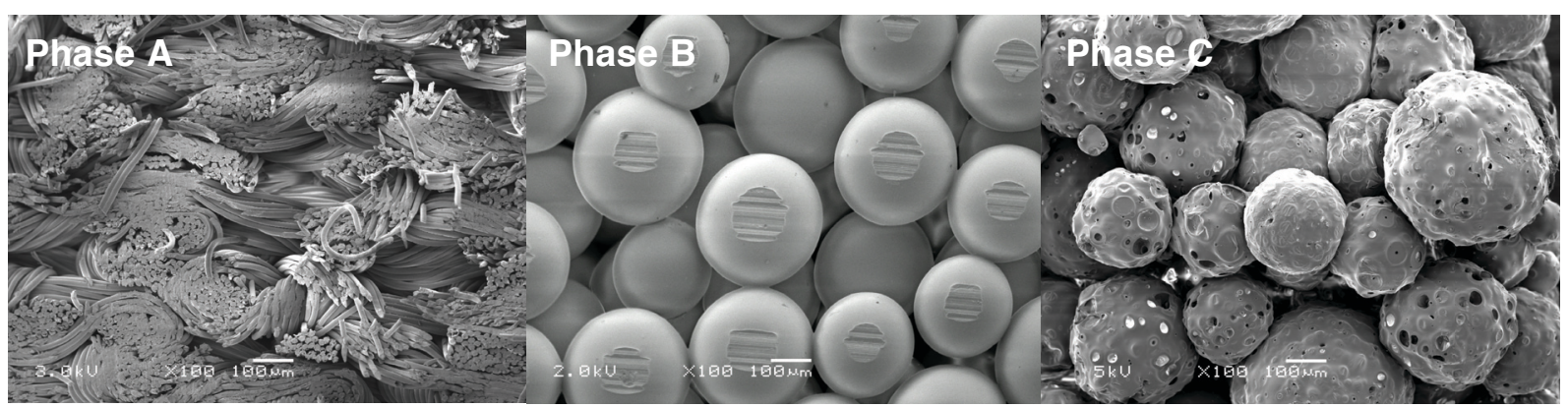

Figure 2. Microstructure of phases A, B, and C of the tri-phasic scaffold.

\section{Acknowledgements}

This work was supported by the National Institutes of Health (R01-AR055280, HHL), Presidential Early Career Award for Scientists and Engineers (PECASE, HHL), the National Science Foundation (Graduate Research Fellowship, JPS) and the New York State Stem Cell ESSC Board (NYSTEM, HHL).

\section{Reference List}

[1] R. J. Johnson. The anterior cruciate: a dilemma in sports medicine. Int.J Sports Med. 3 (1982) 71-79.

[2] American Academy of Orthopaedic Surgeons. Arthoplasty and Total Joint Replacement Procedures: United States 1990 to 1997. (1997). 
[3] C. H. Brown, Jr. and E. W. Carson. Revision anterior cruciate ligament surgery. Clin.Sports Med. 18 (1999) 109-71.

[4] United States Department of Health and Human Services, Centers for Disease Control and Prevention National Center for Health Statistics. National Survey of Ambulatory Surgery. (1996).

[5] United States Department of Health and Human Services, Centers for Disease Control and Prevention National Center for Health Statistics. National Hospital Discharge Survey. (1996).

[6] R. C. Bray, C. A. Leonard, and P. T. Salo. Vascular physiology and long-term healing of partial ligament tears. J.Orthop.Res. 20 (2002) 984-89.

[7] G. R. Barrett, et al. Reconstruction of the anterior cruciate ligament in females: A comparison of hamstring versus patellar tendon autograft. Arthroscopy 18 (2002) $46-54$.

[8] B. D. Beynnon, et al. Anterior cruciate ligament replacement: comparison of bonepatellar tendon-bone grafts with two-strand hamstring grafts. A prospective, randomized study. J Bone Joint Surg Am 84-A (2002) 1503-13.

[9] K. L. Markolf, et al. Effects of femoral tunnel placement on knee laxity and forces in an anterior cruciate ligament graft. J.Orthop.Res. 20 (2002) 1016-24. 
[10] J. C. Loh, et al. Knee stability and graft function following anterior cruciate ligament reconstruction: Comparison between 11 o'clock and 10 o'clock femoral tunnel placement. Arthroscopy 19 (2003) 297-304.

[11] B. Fleming, et al. Effect of tension and placement of a prosthetic anterior cruciate ligament on the anteroposterior laxity of the knee. J.Orthop.Res. 10 (1992) 17786.

[12] R. J. Gregor and T. A. Abelew. Tendon force measurements and movement control: a review. Med.Sci.Sports Exerc. 26 (1994) 1359-72.

[13] B. Beynnon, et al. A sagittal plane model of the knee and cruciate ligaments with application of a sensitivity analysis. J Biomech Eng 118 (1996) 227-39.

[14] B. D. Beynnon, et al. The effect of functional knee bracing on the anterior cruciate ligament in the weightbearing and nonweightbearing knee. Am J Sports Med 25 (1997) 353-59.

[15] B. C. Fleming, et al. The relationship between graft tensioning and the anterior-posterior laxity in the anterior cruciate ligament reconstructed goat knee. J.Orthop.Res. 19 (2001) 841-44.

[16] B. C. Fleming and B. D. Beynnon. In vivo measurement of ligament/tendon strains and forces: a review. Ann.Biomed.Eng 32 (2004) 318-28.

[17] D. B. Robertson, D. M. Daniel, and E. Biden. Soft tissue fixation to bone. Am.J Sports Med. 14 (1986) 398-403. 
[18] M. Kurosaka, S. Yoshiya, and J. T. Andrish. A biomechanical comparison of different surgical techniques of graft fixation in anterior cruciate ligament reconstruction. Am.J Sports Med. 15 (1987) 225-29.

[19] S. A. Rodeo, et al. Use of recombinant human bone morphogenetic protein-2 to enhance tendon healing in a bone tunnel. Am.J Sports Med. 27 (1999) 476-88.

[20] S. A. Rodeo, et al. Tendon-healing in a bone tunnel. A biomechanical and histological study in the dog. J.Bone Joint Surg.Am. 75 (1993) 1795-803.

[21] M. J. Friedman, et al. Autogeneic anterior cruciate ligament (ACL) anterior reconstruction of the knee. A review. Clin Orthop 196 (1985) 9-14.

[22] D. W. Jackson, et al. Cruciate reconstruction using freeze dried anterior cruciate ligament allograft and a ligament augmentation device (LAD). An experimental study in a goat model. Am.J Sports Med. 15 (1987) 528-38.

[23] Yahia, L. Ligaments and Ligamentoplasties. Berlin Heidelberg: Springer Verlag, 1997.

[24] R. R. Cooper and S. Misol. Tendon and ligament insertion. A light and electron microscopic study. J.Bone Joint Surg.Am. 52 (1970) 1-20.

[25] M. Benjamin, E. J. Evans, and L. Copp. The histology of tendon attachments to bone in man. J.Anat. 149 (1986) 89-100.

[26] X. Wei and K. Messner. The postnatal development of the insertions of the medial collateral ligament in the rat knee. Anat.Embryol.(Berl) 193 (1996) 53-59. 
[27] Visconti C. Sagarriga, et al. Biochemical analysis of collagens at the ligament-bone interface reveals presence of cartilage-specific collagens. Arch.Biochem.Biophys. 328 (1996) 135-42.

[28] C. Niyibizi, et al. Identification and immunolocalization of type X collagen at the ligament-bone interface. Biochem.Biophys.Res Commun. 222 (1996) 584-89.

[29] K. Messner. Postnatal development of the cruciate ligament insertions in the rat knee. morphological evaluation and immunohistochemical study of collagens types I and II. Acta Anatomica. 160 (1997) 261-68.

[30] W. Petersen and B. Tillmann. Structure and vascularization of the cruciate ligaments of the human knee joint. Anat.Embryol.(Berl) 200 (1999) 325-34.

[31] S. Thomopoulos, et al. Variations of biomechanical, structural, and compositional properties along the tendon to bone insertion site. J Orthop Res 21 (2003) 413-19.

[32] I. E. Wang, et al. Age-dependent changes in matrix composition and organization at the ligament-to-bone insertion. J.Orthop.Res. 24 (2006) 1745-55.

[33] S. L. Woo and J. A. Buckwalter. AAOS/NIH/ORS workshop. Injury and repair of the musculoskeletal soft tissues. Savannah, Georgia, June 18-20, 1987. J.Orthop.Res. $6(1988) 907-31$.

[34] J. P. Spalazzi, et al. In vivo evaluation of a multiphased scaffold designed for orthopaedic interface tissue engineering and soft tissue-to-bone integration. J.Biomed.Mater.Res.A. 86 (2008) 1-12. 
[35] J. P. Spalazzi, et al. Development of controlled matrix heterogeneity on a triphasic scaffold for orthopedic interface tissue engineering. Tissue Eng 12 (2006) 3497508.

[36] M. Borden, et al. Tissue engineered microsphere-based matrices for bone repair: design and evaluation. Biomaterials 23 (2002) 551-59.

[37] I. E. Wang, et al. Role of osteoblast-fibroblast interactions in the formation of the ligament-to-bone interface. J.Orthop.Res. 25 (2007) 1609-20.

[38] H. H. Lu, et al. Anterior cruciate ligament regeneration using braided biodegradable scaffolds: in vitro optimization studies. Biomaterials 26 (2005) 4805-16. 